\title{
Distress, demoralization and psychopathology: Diagnostic boundaries
}

\author{
John M. de Figueiredo, MD, ScD \\ Yale University School of Medicine
}

USA

\begin{abstract}
Background and Objectives: The objectives of the manuscript are: (a) to review the understanding of demoralization and its assessment; (b) to describe its clinical progression; (c) to explain the differences between demoralization and other form of psychological distress; (d) to propose a set of criteria for future research on demoralization.

Methods: A MEDLINE search using the keywords distress, subjective incompetence, depression, demoralization, helplessness, hopelessness and psychopathology was conducted. This was supplemented by a manual search of the literature.

Results: Demoralization can be distinguished from passing or transient distress, nonspecific distress, sub-threshold depression or anxiety, and certain mental disorders. Demoralization can be a risk factor for the manifestation of psychopathology, the prodromal phase of a mental disorder, or a trigger for exacerbation or recurrence of psychiatric distress symptoms. The domains of distress and demoralization are described and research diagnostic criteria for demoralization are presented.

Conclusions: The scales discussed in this article differ in their time frames and have not yet been applied to the same population at the same time. The role of demoralization as a risk factor for mental disorders is just beginning to be understood. The domains and the diagnostic criteria for demoralization presented in this article need to be confirmed by epidemiological and empirical studies. Future research should continue to clarify its role in the pathogenesis of both mental disorders and physical illnesses and identify appropriate interventions for its arrest or prevention.
\end{abstract}

Received: 15 October 2012

Revised: 10 December 2012

Accepted: 19 December 2012 


\section{Objectives}

This article has the following objectives: (a) to review the evolving understanding of demoralization and the methods proposed for its assessment; (b) to describe the clinical progression of demoralization; (c) to explain the differences between demoralization and passing or transient distress, non-specific distress and sub-threshold depression or anxiety, as well as the differences between demoralization and mental disorders such as major depressive disorder, dysthymic disorder, acute stress disorder, posttraumatic stress disorder, and adjustment disorder; and (d) to propose a set of criteria that may be considered for future research on demoralization.

\section{Understanding demoralization}

As defined by Frank, demoralization is the state of mind of a person deprived of spirit or courage, disheartened, bewildered, and thrown into disorder or confusion. He proposed that this state of mind occurs in many persons who seek psychotherapy, whatever their diagnostic label ${ }^{1,2}$. Demoralization always takes place within the context of a past, present, anticipated or imagined stressful situation. To further characterize the concept of demoralization, it has been proposed that the clinical hallmark of demoralization is "subjective incompetence" (SI) and that demoralization involves both SI and symptoms of distress, such as depression, anxiety, resentment, anger, or combinations thereof ${ }^{3,4}$. SI is a self-perceived incapacity to perform tasks and express feelings deemed appropriate in a stressful situation, resulting in pervasive uncertainty and doubts about the future. The stressful situation disconfirms assumptions about self and others and about the continu- ity of the past and present with the future. Individuals with SI are puzzled, indecisive, uncertain, facing a dilemma, unclear as to ways out of the situation, placed in a deadlock, impasse, quandary, or plight. Having lost the cognitive map, a person with SI has no guide, chart or hint to get out of the predicament. By generalizing and modifying slightly a definition proposed for cancer patients, distress may be defined as follows: "An unpleasant emotional experience of a psychological, social, and/or spiritual nature that may interfere with the ability to cope effectively with a stressful situation. Distress extends along a spectrum, ranging from common non-pathological feelings of vulnerability, sadness, and fears to problems that can become disabling, such as depression, anxiety, panic, social isolation, and spiritual crisis" ${ }^{\prime 5,6}$. Distress may be "specific", i.e. part of a diagnosable mental disorder or physical illness, or "non-specific". SI and distress co-occur when perceived stress is high and/or social support is weak. The combination of SI with depression or other forms of nonspecific or specific distress constitutes demoralization ${ }^{3,4}$. Demoralization is more likely to occur when the stressful situation is relevant to the self-esteem of the demoralized person. The co-occurrence of SI and distress (i.e., demoralization) involves a fundamental change in the person's expectations (subjective likelihoods) and assumptions (subjective certainties), i.e., in the person's "Umwelt" (ambient world) 3 ,

A major advance was the convergence of Frank's clinical observations with Dohrenwend's interpretation of the common dimension measured by psychiatric screening scales as non-specific psychological distress or something akin to demoralization ${ }^{8}$. Dohrenwend noted that the content of the psychiatric screening scales, including a scale he and his colleagues had developed, was very similar to Frank's descriptions of the complaints 
of patients seeking outpatient psychotherapy. $\mathrm{He}$, therefore, referred to his scale as "demoralization scale". Frank had already noted that same or similar complaints had been previously observed in different clinical settings and received different names. For example, a type of existential despair or loss of "fighting spirit" had been noticed by Engel in patients with medical illness and called "giving up-given up complex"9,10; acute demoralization was recognized by Caplan in the emergency departments and called "crisis" and chronic demoralization had been seen in patients with schizophrenia by Gruenberg who called it "social breakdown syndrome"12.

Although demoralization always involves distress, not all non-specific distress is demoralization. Dohrenwend argued that the degree to which an individual experiences nonspecific distress may be considered his or her "psychological temperature" and may provide a conceptual continuity of depressive symptoms from normalcy to clinical disorders ${ }^{8}$. Like inflammation, the distress component of demoralization can be non-specific, may or may not resolve itself if the stressor is removed, and may start as a non-pathological reaction of an individual to the stressor, but become a pathological process or state and require intervention under certain conditions.

\section{Assessment of demoralization}

Five scales have been specifically designated as measuring demoralization and used in research: Dohrenwend's Psychiatric Epidemiology Research Interview - Demoralization Scale [PERI-D], a scale developed by Stewart et al., Kissane's Demoralization Scale [KDS], MMPI-2 Restructured Clinical [RC] Scale of Demoralization [RCd]) and a scale based on and the "Diagnostic Criteria for Psychosomatic Research" (DCPR) ${ }^{8,14-17}$. Recently a scale to measure subjective incompetence was used in a study of outpatients with cancer ${ }^{18,19}$. With these methods in place, a number of studies conducted in the United States, Canada, Europe, Israel, Australia and New Zealand have documented demoralization in both clinic populations and community samples. Of particular interest has been the demonstration that demoralization can be distinguished from depression among refugees and immigrants ${ }^{2}$.

PERI-D, KDS, RCd and DCPR seem to measure somewhat different aspects or different stages of demoralization. The PERI-D appears to measure primarily non-specific distress, does not have any exclusionary criteria and can be either a trait or a state measure, depending on the time-set and the circumstances in which the measure is taken. For Dohrenwend ${ }^{8}$, demoralization is a condition of low self-esteem, helplessness, hopelessness, sadness, and anxiety. Stewart et al. ${ }^{15}$, defined demoralization as a perception by the patients that their ability to positively affect their own future is too likely to be ineffectual to warrant efforts at change and developed a scale by selecting from the Beck Depression Inventory, the Beck Hopelessness Scale, and the Beck Dysfunctional Attitudes Scale items they believed were indicative of demoralization. KDS is a state measure. Clarke and Kissane proposed the following six criteria for the diagnosis of demoralization: "1) affective symptoms of existential distress, including hopelessness or loss of meaning and purpose in life; 2) cognitive attitudes of pessimism, helplessness, sense of being trapped, personal failure, or lacking a worthwhile future; 3 ) conative absence of drive or motivation to cope differently; 4) associated features of social alienation or isolation and lack of support; 5) allowing for fluctuation in emotional intensity, these phe- 
nomena persist across more than 2 weeks; 6 ) a major depressive or other psychiatric episode is not present as the primary condition" 20,21 . The RCd is a scale obtained by extracting an overarching factor that was common to the original 10 clinical scales of the MMPI ${ }^{16}$. The DCPR criteria describe demoralization as a "feeling state characterized by the patient's consciousness of having failed to meet his or her own expectations (or those of others) or being unable to cope with some pressing problems; the patient experiences feelings of helplessness, or hopelessness, or giving up; these feelings occur before the manifestation of a physical disorder or exacerbate that disorder". Furthermore, the criteria require that the feeling be "prolonged or generalized (at least one month duration)"13,14.

\section{The clinical progression of demoralization}

As the intensity or duration of the stressful situation increases, SI eventually becomes helplessness. This happens when the patient develops expectations of being personally unable to change the likelihood of having a positive (i.e., highly desired) outcome or avoiding a negative outcome but still hopeful that the circumstances will change. In some cases, an individual who is helpless eventually becomes hopeless, i.e., certain that a positive outcome will not take place or a negative outcome will take place. This cascade of events may culminate in existential despair, meaninglessness and suicide. As Abramson and her colleagues noted, hopelessness always involves helplessness, i.e., hopelessness is a subset of helplessness, and, therefore, when hopelessness occurs, helplessness also occurs 22 (Fig 1).
Helplessness is not listed or required in DSM-IV-TM for the diagnoses of major depressive episode, dysthymic disorder, or adjustment disorder with depressed mood. Hopelessness is listed in DSM-IV-TM as a manifestation of dysthymic disorder, but research has shown that hopelessness is a mental state that is distinct from depression ${ }^{23,24}$. When measured with the Beck Hopelessness Scale, hopelessness has been shown among psychiatric patients to be a stronger predictor of suicide than depression ${ }^{25,26}$. In advanced medical illness, hopelessness is associated with demoralization, impaired spiritual wellbeing and poor quality of life $\mathrm{f}^{17,27,28}$. This is consistent with the view that SI, and not distress, is the clinical hallmark of demoralization $^{3}$. The occurrence of hopelessness implies a drastic change in the assumptive world ("Umwelt") of the patient ${ }^{3,7}$.

Demoralization is a spectrum or a gradient that starts with SI and non-pathological distress. SI becomes helplessness, sometimes grows into hopelessness, and becomes pathological when it is enduring and causes significant impairment in important areas of functioning. In its most severe form, demoralization can be recognized as a syndrome, including both SI-helplessness-hopelessness and distress that may be non-specific or specific (i.e., part of a physical illness or another mental disorder). The psychiatric outpatients studied by Frank were probably in the milder range of the demoralization spectrum, while those in a state of crisis described by Caplan and those with medical illness identified by Engel probably displayed a more severe form in this spectrum ${ }^{1,9-11}$. At a time when treatment strategies were not as advanced as they are today, the accumulation of frustrations experienced by patients with schizophrenia probably led to a chronic form of demoralization, the so-called "social breakdown syndrome", identified as a diagnosis in DSM- 


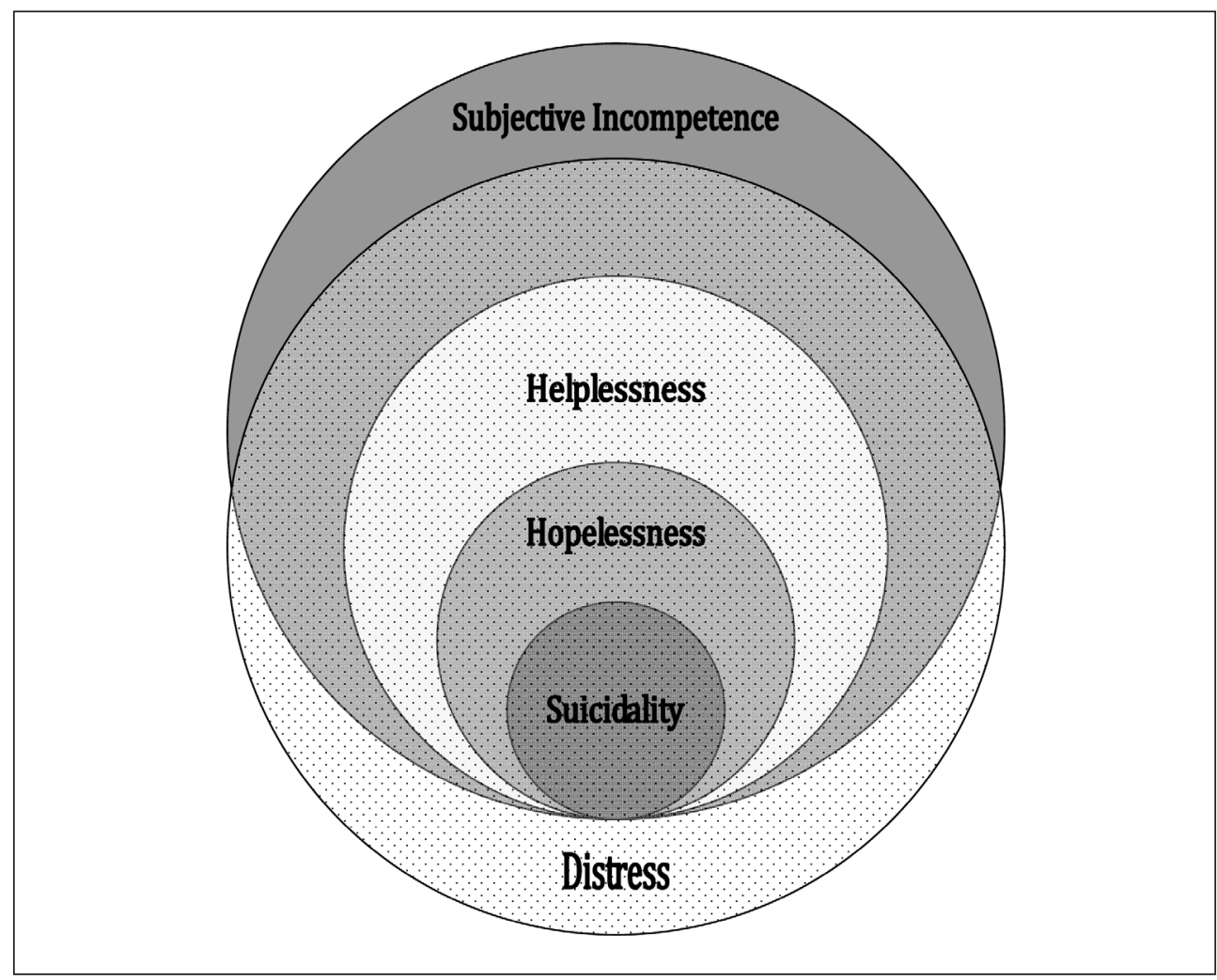

Figure 1.

$\mathrm{II}^{12}$. Kissane and his colleagues proposed that a "demoralization syndrome be recognized as a distinct psychiatric disorder in which loss of meaning and hope can potentially spoil any sense of a worthwhile life and future" ${ }^{20}$. Clarke and Kissane also reminded us that demoralization is not a static entity but a process ${ }^{21}$. Clinical observations capture this process at various phases of presentation and, in the absence of biological markers, thresholds or "cut-off points" are established to define what constitutes a disorder, subject to further review, as new research findings are reported.

\section{Differential diagnosis}

Demoralization should be distinguished from passing or transient distress, non-specific distress and sub-threshold depression or anxiety. It should also be distinguished from certain mental disorders such as major depressive disorder, dysthymic disorder, acute stress disorder, posttraumatic stress disorder, and adjustment disorder. The understanding of these differences is important not only for the development and implementation of meaningful treatment interventions but also for the clarification of the prognosis. 


\section{Passing or transient distress or non-specific distress}

The distinction between "distress" and "demoralization" is at the center of a debate whether demoralization is a "normal" (i.e., non-pathological) or homeostatic (and, therefore, not requiring any intervention) or "abnormal" (i.e., pathological) reaction to adversity $^{29-31}$. In a community-based sample, the prevalence of demoralization as measured by DCPR was far less frequent than the rates found among medically ill patients, suggesting that demoralization does not simply identify generic psychological distress ${ }^{32}$. Distress may get better with medication, psychotherapy, both or doing nothing. The cooccurrence of SI converts distress into demoralization ${ }^{4,7}$. Non-pathological grief is an example of distress without SI and can be distinguished from both demoralization and anhedonic depression ${ }^{33,34}$. SI may occur without distress, as might happen with a recent immigrant excited by his land of promise or an employee rewarded by an unexpected promotion.

\section{Sub-threshold depression or anxiety}

The distress expressed by the symptoms of sub-threshold depression or anxiety (i.e., depression or anxiety that does not meet the DSM-IV-TM criteria for major depressive disorder, dysthymic disorder, generalized anxiety disorder or some other diagnostic category involving depression or anxiety) is not the same as demoralization. Such symptoms may occur without a co-occurrence of SI and, therefore, they would not, by themselves, meet the criteria for the definition of demoralization. The symptoms subsumed as "nonspecific distress" or "sub-threshold de- pression or anxiety" may be part of demoralization. However, demoralization also involves SI. A study of outpatients with cancer revealed that sub-threshold depression and SI occur separately when perceived stress is low and social support is high ${ }^{19}$.

\section{Adjustment disorder}

Demoralized individuals are sometimes incorrectly diagnosed as having "adjustment disorder" or "depressive disorder, not otherwise specified", largely because demoralization is not a diagnosis in DSM-IV-TM. According to DSM-IV-TM, "adjustment disorder" is an exclusion diagnosis, requires an arbitrary judgment that the distress is in excess of what would be expected from exposure to the stressor and the diagnosis cannot even be made without verification that the symptoms do not persist for more than 6 months once the stressor, or its consequences, have terminated. Such criteria are not required for demoralization, and, therefore, it is incorrect to confuse demoralization with adjustment disorder. A recent study found the diagnosis of adjustment disorder to have inconsistent clinical description and prognosis, inadequate differentiation from other disorders, and devoid of specific psychometric and neurobiological features. The authors concluded that the spectrum of mood disturbances entailed by the diagnosis of adjustment disorder appears to be too broad. A major problem seems to lie in the fact that it is an exclusion diagnosis that overlaps with sub-threshold manifestations of mood and anxiety disorders ${ }^{35}$. The distinction between demoralization and adjustment disorder was demonstrated in a study of medically ill patients, where demoralization according to DCPR occurred only in one third of those with a DSM-IV diagnosis of adjustment disorder ${ }^{36}$. 


\section{Acute stress disorder and posttraumatic stress disorder}

Demoralization, acute stress disorder (ASD) and posttraumatic stress disorder (PTSD) are all related to adaptation to stress. Criteria for the diagnosis of PTSD were delineated for the first time in 1980 in DSM-III. In 1995 the diagnosis of ASD was added to DSM-IV and later to DSM-IV-TM. By definition, a diagnosis of ASD is appropriate only for symptoms that occur within one month of the stressor. PTSD requires more than one month of symptoms, and, therefore, the diagnosis of PTSD cannot be made during this one month period. Since all three conditions require exposure to a stressful situation and their clinical manifestations may include both helplessness (an advanced form of SI) and psychological distress, it is of the utmost importance not to confuse demoralization with ASD and PTSD.

Not all stressful situations qualify for the diagnosis of ASD and PTSD. According to DSM-IV-TM, this diagnosis requires that the person had been exposed to a traumatic event or events that involved actual or threatened death or serious injury, or a threat to the physical integrity of self or others. This is to be contrasted with demoralization which requires only a past, present, anticipated or imagined stressful situation. For individuals who have an extreme stressor but who develop a symptom pattern that does not meet criteria for ASD, DSM-IV-TM recommends that the diagnosis of adjustment disorder be considered, thus introducing for this population the difficulties associated with this diagnosis mentioned above. Furthermore, both ASD and PTSD require the presence of several other symptoms, such as dissociative symptoms, re-experiencing of the traumatic event, avoidance of the recollection of the trauma, numbing of general responsiveness, and increased arousal. None of these symp- toms or behaviors is required for the identification of demoralization. Nevertheless, since it is important not to miss the diagnosis of ASD, and until research discovers new methods for differential diagnosis, a clinical presentation of helplessness and distress should not be designated as demoralization if it is better accounted for by ASD. Demoralization could be co-morbid with either ASD or PTSD, however. If a person is overwhelmed by a feeling of helplessness (an advanced state of SI), he or she may become demoralized in response to the relentless intrusion of post-trauma symptoms. Recently demoralization was found to be a risk factor for PTSD ${ }^{37,38}$.

\section{Dysthymic disorder}

According to DSM-IV-TM, dysthymic disorder is characterized by at least two years of depressed mood for more days than not, accompanied by additional depressive symptoms that do not meet criteria for a major depressive episode. With one exception, dysthymic disorder does not share with demoralization the presence of SI (or its more advanced form, helplessness). The single exception is hopelessness which is only one of six symptoms of which two are required for the diagnosis, in addition to depressed mood. As Abramson noted, however, hopelessness always involves helplessness, i.e., hopelessness is a subset of helplessness ${ }^{22}$. If hopelessness is one of the symptoms included for the diagnosis of dysthymic disorder, then the patient is also demoralized.

\section{Major depressive disorder}

According to Schildkraut and Klein, anhedonia is the distinctive feature of major depressive disorder, while demoralized patients experience a sense of inefficacy in dealing 
with a stressful situation rather than anhedonia $^{39}$. According to Klein, demoralization should not be confused with what he called "endogenomorphic depression", a construct that maps closely onto the category of "major depressive disorder with melancholia" in DSM-III. He proposed that the core process in endogenomorphic depression is impairment in the capacity to experience pleasure. In particular, demoralization, according to Klein, involves a loss of anticipatory pleasure but not of consummatory pleasure, while in major depressive disorder both anticipatory and consummatory pleasures are lost $\mathrm{t}^{40,41}$. It is unclear how this distinction would handle cases in which individuals with major depressive disorder are also demoralized.

Demoralization can be distinguished from clinical depression (i.e., depression that meets the DSM criteria for a diagnosable entity) by examining the motivation of the patient ${ }^{7}$. Motivation may be viewed as a vector, involving a magnitude and a direction. SI is the loss of the directional component of motivation. Demoralization starts as uncertainty about the direction one's actions should take, even though the magnitude of motivation is intact. In clinical depression, however, the magnitude of motivation is reduced from the beginning, even when the direction of action is known. Some patients with depression may retain the magnitude of motivation but lose the sense of direction; others may retain the sense of direction but lose the magnitude of motivation; and still others may lose both the magnitude and the direction. We may postulate that the first group is demoralized but not clinically depressed; the second is clinically depressed but not demoralized; and the third is both demoralized and clinically depressed. These phenomenological observations have been confirmed by a study on the characterization of psychopathology in patients with medical illnesses. Using their scale together with other scales, Kissane and colleagues identified sub-groups of cancer patients who scored high in the KDS but had low scores for depression as a form of nonspecific distress, for anhedonic depression and for major depressive disorder ${ }^{20}$. Clarke and colleagues, using latent trait analysis, found that the dimension of demoralization can be separated from that of anhedonic depression $^{42,43}$. Similar observations have been made with cancer patients ${ }^{30}$. In a study of monozygotic twins discordant for a lifetime history of major depression, one of the three clusters of variables that were significantly different between affected and non-affected twins included higher scores in the twins with major depression in "acting out", in alcohol dependence and in attributes that may be viewed as surrogate (proxy) indicators of SI-helplessness, such as powerlessness, lack of self-efficacy, and a diminished self-perceived sense of mastery ${ }^{44}$.

The application of the DCPR revealed that it is both possible and clinically necessary to distinguish demoralization from major depressive disorder ${ }^{13,14}$. In a multicenter study involving outpatients recruited from different medical settings (gastroenterology, cardiology, endocrinology and oncology), a group of researchers examined the prevalence and overlap rates between demoralization according to the DCPR and DSM-IV major depressive disorder. Demoralization was identified in $30.4 \%$ of patients, while major depression was present in $16.7 \%$. There was a considerable overlap between the two diagnoses. However, $43.7 \%$ patients with major depressive disorder were not demoralized, and $69 \%$ of demoralized patients did not satisfy the criteria for major depressive disorder. The findings suggest that demoralization is quite prevalent among the medically ill and that it is distinct and not hierarchically related to major depressive disorder ${ }^{45}$. The dis- 
tinction between demoralization and major depressive disorder was confirmed in other clinical populations ${ }^{46}$, including patients with skin diseases ${ }^{47}$ and consultation-liaison psychiatry patients ${ }^{48}$. In a sample of heart transplanted patients, demoralization was significantly associated with a decrease in specific dimensions of psychological well-being and the co-occurrence of major depressive disorder did not alter those associations ${ }^{49}$.

\section{Discussion}

This article addresses the problem of recognizing demoralization as it advances in severity and drawing the boundaries between demoralization and certain mood and anxiety disorders. The clinical relevance of this problem is obvious. The problem of diagnosis of demoralization is complicated, partly because of the need for a clear distinction between distress and SI, partly because demoralization appears to be a process and not a static and immutable entity, and partly because demoralization may coexist with mental disorders as well as physical illnesses. For example, demoralization has been documented in primary care patients ${ }^{45,46}$, and in patients with coronary artery disease ${ }^{50}$, motor neuron disease $^{51}$, functional gastrointestinal disorders $^{52,53}$, breast cancer ${ }^{54,55}$, and gastrointestinal and colorectal cancer ${ }^{19,45,46}$. Hopelessness was significantly associated with an increased risk of cardiovascular disorders and cancer ${ }^{56-60}$, predicted the length of survival in patients with coronary artery disease ${ }^{61}$ and the progression of carotid atherosclerosis ${ }^{62}$.

Demoralization is a risk factor for mental or physical morbidity, including suicide; a prodromal phase of many mental disorders; and a trigger for an ensuing episode of major depressive disorder ${ }^{45,46}$. Just as confusion of demoralization with nonspecific distress, adjustment disorder or major depressive disorder has the potential for harming patients, so do the "under-recognition" of demoralization, as in patients with terminal illnesses, and its "over-recognition", as in patients with depression associated with stroke.

The criteria proposed by Clarke and Kissane do not clearly distinguish between distress and SI or how to handle the co-occurrence of demoralization and ASD or dysthymic disorder ${ }^{20,21}$. The DCPR criteria were designed for research on psychosomatic disorders but demoralization can co-occur with other forms of psychopathology as well ${ }^{13,14}$. Due to these limitations, future research on demoralization should consider a new set of diagnostic criteria (Table 1).

The diagnosis of demoralization has important therapeutic and prognostic implications. The scenario described above regarding PTSD could take place with other mental disorders when the symptoms of those disorders are so persistent and severe they become a source of demoralization (e.g., hallucinations in schizophrenia). This complicated vicious circle might prolong the recovery from the other disorder, having probably created, in the case of schizophrenia, years ago, in Gruenberg's time, a type of chronic demoralization he had called "social breakdown syndrome" 12 . It has been observed that tapering of antidepressant medications in patients with stabilized non-acute schizophrenia may not improve the outcome of antipsychotic monotherapy; such patients were probably demoralized and psychotherapy might have been more useful ${ }^{63}$. In a study of patients with major depressive disorder, after controlling for depression severity at baseline, a greater degree of hopelessness was found to significantly increase the risk for non-response to fluoxetine as well as the risk of greater endpoint depression severity ${ }^{64}$. In ad- 
Table 1

Research Diagnostic Criteria for Demoralization

All six criteria must be met:

(A) The presence of subjective incompetence-helplessness-hopelessness: Helplessness is indicated by subjective account or observation by others of expectations of lowered ability for changing the likelihood of a highly unwanted outcome taking place or a highly wanted outcome not taking place. Examples of symptoms and behaviors characteristic of helplessness are the following: belief of lack of ability to control what happens; belief of inability to attain a successful future due to a sense of being trapped; loss of sense of continuity between the past and the future; conviction of being unable to meet his or her own assumptions or expectations, or those of others. In mild cases, the patient experiences subjective incompetence, i.e., a self-perceived incapacity to perform tasks and express feelings deemed appropriate in a stressful situation, resulting in pervasive uncertainty and doubts about the future. Examples of symptoms of subjective incompetence are being puzzled, indecisive, uncertain, facing a dilemma, unclear as to ways out of the situation, placed in a deadlock, impasse, quandary, or plight. In extreme cases, the patient also experiences hopelessness, i.e., certainty that a highly unwanted outcome will take place or a highly wanted outcome will not take place.

(B) The presence of distress as indicated by subjective account or observation by others of one or more of the following: depressed mood (sad affect), anxiety, anger, shame, social isolation and spiritual crisis.

(C) The symptoms always take place within the context of a past, present, anticipated or imagined stressful situation.

(D) The symptoms cause significant impairment in social, occupational, educational, or other important areas of functioning.

(E) The symptoms are not due to the direct physiological effects of a substance (e.g., a medication or a drug of abuse) or a general medical condition; and are not merely an exacerbation of a preexisting Axis I or Axis II disorder. If, however, a person is overwhelmed by a feeling of helplessness, they may become demoralized in response to the relentless distress of symptoms of another mental disorder (e.g., intrusive post trauma symptoms in PTSD or severe hallucinations in schizophrenia) in which case both demoralization and the other disorder should be diagnosed.

(F) The symptoms describe above in criteria (A) through (E) occur for most of the day and for more days than not; if they occur for two weeks or less, the diagnosis of possible demoralization should be made; if they occur for more than two weeks, the diagnosis of probable demoralization should be made.

Some of the symptoms given in criterion (B) occur in other disorders but they do not, by themselves, justify the diagnosis of demoralization. Certain symptoms specific of those other disorders (e.g., anhedonia in major depressive disorder) or non-specific (e.g., disturbance of sleep, appetite or body weight) are not part of demoralization.

dition to genetic predisposition, developmental history, personality traits, perceived stress, perceived social support and cultural factors, demoralization stands out as a risk factor for the manifestation of psychopathology and certain physical illnesses, the prodromal phase of a mental disorder, or a trigger for exacerbation or recurrence of psychiatric distress symptoms. It follows logically that relief of demoralization comes from the separation of its two components, distress and SI-helplessness-hopelessness, or from the relief of at least one of those two components or preferably both.

The limitations of the conclusions should be recognized. The five scales of demoralization, the SI scale, and scales to assess helplessness and hopelessness have not yet been applied to the same population at the same time. It is thus conceivable, for example, that some people who do not meet the DCPR cri- 
teria might score high in the PERI-D or KDS or vice-versa. The scales differ in their time frames. The role of demoralization as a risk factor for mental disorders is just beginning to be understood and more prospective studies are needed to better define this role. This article presents domains of distress and demoralization and research diagnostic criteria for demoralization. The proposed domains and criteria need to be confirmed by epidemiological and empirical studies.

\section{Conclusion}

To conclude, research has established the widespread presence of demoralization in a number of clinical contexts and in the general population, its correlation with a past, present, anticipated or even imagined stressful situation and its potential for increasing the vulnerability to ill-health. Future research should continue to clarify its role in the pathogenesis of both mental disorders and physical illnesses and identify appropriate interventions for its arrest or prevention.

\section{Acknowledgement}

The author thanks the following colleagues for their support and helpful comments on earlier versions of this manuscript: Barton J. Blinder MD, David M. Clarke MBBS, Paul Desan MD, Julia Frank MD, James L. Griffith MD, Ramakrishnan Shenoy MD, Robert Kohn MD, Roger Peele, MD, Jerome Rogoff MD, Ramaswamy Viswanathan MD, Thomas N. Wise MD and Sara Gostoli MA. The author also thanks Giovanni A. Fava MD, Bruce P. Dohrenwend Ph.D. and Laura Sirri Psy.D. for providing him with helpful information during the preparation of this article. Mr. Nathan Molina was helpful in the preparation of the illustration.

\section{References}

1. Frank JD, Frank JB. Persuasion and Healing, A Comparative Study of Psychotherapy. Baltimore and London: The Johns Hopkins University Press; 1991.

2. de Figueiredo JM. Demoralization and psychotherapy: A tribute to Jerome D. Frank, MD, PhD. (1909-2005). Psychother Psychosom 2007; 76(3): 129-133.

3. de Figueiredo JM, Frank JD. Subjective incompetence, the clinical hallmark of demoralization. Compr Psychiatry $1982 ; 23(4): 353-363$.

4. de Figueiredo JM. Deconstructing demoralization: Subjective incompetence and distress in the face of adversity. In: Alarcon RD, Frank JB, editors. The Psychotherapy of Hope: The Legacy of Persuasion and Healing. Baltimore, MD: The Johns Hopkins University Press; 2012. p. 107-124.

5. National Comprehensive Cancer Network. Distress Management Clinical Practice Guidelines in Oncology. JNCCN 2003; 1: 344-374.

6. Jacobsen P, Donovan K, Trask M, Fleishman S, Zabora J, Baker F, et al. Screening for psychologic distress in ambulatory cancer patients: a multicenter evaluation of the distress thermometer. Cancer 2005; 103(7): 1494-1502.

7. de Figueiredo JM. Depression and demoralization: phenomenologic differences and research perspectives. Compr Psychiatry 1993; 34: 308-311.

8. Dohrenwend BP, Shrout PE, Egri G, Mendelsohn FS. Non-specific psychological distress and other measures for use in the general population. Arch Gen Psychiatry 1980; 37 : 1229-1236

9. Engel GL. A psychological setting of somatic disease: the "giving up-given up complex". Proc Roy Soc Med 1967; 60: 553-555.

10. Engel GL. A life setting conducive to illness, the giving up-given up complex. B Menninger Clin 1968; 32: 355-365.

11. Caplan G. Principles of Preventive Psychiatry. New York, NY: Basic Books; 1964.

12. Gruenberg EM. The social breakdown syndrome and its prevention. In: Caplan G, editor. American Handbook of Psychiatry. New York, NY: Basic Books; 1974. p. 697-711. 
13. Fava GA, Freyberger HJ, Bech P, Christodoulou G, Sensky T, Theorell T, et al. Diagnostic criteria for use in psychosomatic research. Psychother Psychosom 1995; 63(1): 1-8.

14. Fabbri S, Fava GA, Sirri L, Wise TN. Development of a New Assessment Strategy in Psychosomatic Medicine: the Diagnostic Criteria for Psychosomatic Research. In: Porcelli P, Sonino N, editors. Psychological Factors Affecting Medical Conditions. A New Classification for DSM-V. Advances in Psychosomatic Medicine 28. Basel: Karger; 2007; p. 1-20.

15. Stewart JW, Mercier MA, Quitkin FM, McGrath PJ, Nunes E, Young J, et al. Demoralization predicts nonresponse to cognitive therapy in depressed outpatients. J Cognitive Psychother 1993; 7: 105-116.

16. Tellegen A, Ben-Porath YS, McNulty JL, Arbisi PA, Graham JR, Kaemmer B. The MMPI-2 Restructured Clinical (RC) Scales: Development, Validation, and Interpretation. Minneapolis, MN: University of Minnesota Press; 2003.

17. Kissane DW, Wein S, Love A, Lee XO, Kee PL, Clarke DM. The Demoralization Scale: a report of its development and preliminary validation. J Palliat Care 2004; 20(4): 269-276.

18. Cockram C, Doros G, de Figueiredo JM. Diagnosis and measurement of subjective incompetence, the clinical hallmark of demoralization. Psychother Psychosom 2009; 78: 342-345.

19. Cockram C, Doros G, de Figueiredo JM. Subjective incompetence as the clinical hallmark of demoralization in cancer patients without mental disorder. Primary Psychiatry 2010; 17(7): 54-58.

20. Kissane D, Clarke D, Street A. Demoralization syndrome, a relevant psychiatric diagnosis for palliative care. $\mathrm{J}$ Palliat Care 2001; 17: 12-21.

21. Clarke DM, Kissane DW. Demoralization: its phenomenology and importance. Aust N Z J Psychiatry 2002; 36: 733-742.

22. Abramson LY, Metalsky GI, Alloy LB. Hopelessness Depression: A theory-based sub-type of depression. Psychol Rev 1989; 962: 358-372.

23. Glanz LM, Haas GL, Sweeney JA. Assessment of hopelessness in suicidal patients. Clin Psychol Rev 1995; 15 : 49-64.

24. Shahar G, Bareket L, Rudd MD, Joiner TE. In severely suicidal young adults, hopelessness, depressive symptoms, and suicidal ideation constitute a single syndrome. Psychol Med 2006; 36: 913-922.

25. Wetzel RD, Margulies T, Davis R. Hopelessness, depression, and suicide intent. J Clin Psychiatry 1980; 41: 159160 .
26. Beck AT, Steer RA, Beck JS, Newman CF. Hopelessness, depression, suicidal ideation, and clinical diagnosis of depression. Suicide Life-Threat 1993; 23: 139-145.

27. Sullivan MD. Hope and hopelessness at the end of life. Am J Geriatr Psychiatry 2003; 11: 393-405.

28. McLeod JE, Clarke DM. A review of psychosocial aspects of motor neuron disease. J Neurol Sci 2007; 258: 4-10.

29. Slavney PR. Diagnosing demoralization in consultation psychiatry. Psychosomatics 1999; 40(4): 325-329.

30. Angelino AF, Treisman GJ. Major depression and demoralization in cancer patients: diagnostic and treatment considerations. Support Care Cancer 2001; 9: 344-349.

31. Griffith JL, Gaby L. Brief psychotherapy at the bedside: countering demoralization from medical illness. Psychosomatics 2005; 46(2): 109-116.

32. Mangelli L, Semprini F, Sirri L, Fava GA, Sonino N. Use of the Diagnostic Criteria for Psychosomatic Research (DCPR) in a community sample. Psychosomatics 2006; 47 : 143-146.

33. Clarke DM, Kissane DW, Trauer T, Smith GC. Demoralization, anhedonia and grief in patients with severe physical illness. World Psychiatry 2005; 4: 96-105.

34. Wellen M. Differentiation between demoralization, grief and anhedonic depression. Curr Psych Rep 2010; 12(3): 229-233.

35. Semprini F, Fava GA, Sonino N. The spectrum of Adjustment of Disorders: too broad to be clinically helpful. CNS Spectrum 2010; 15(6): 382-388.

36. Grassi L, Mangelli L, Fava GA, Grandi S, Ottolini F, Porcelli P, et al. Psychosomatic characterization of adjustment disorders in the medical setting: some suggestions for DSM-V. J Affect Disorders 2007; 101: 251-254.

37. Kohn R, Levav I, Donaire I, Machuca M, Tamashiro R. Psychological and psychopathological reactions in Honduras following Hurricane Mitch: implications for service planning. Revista Panamericana de Salud Publica 2005; 18(4/5): 287-295.

38. Kohn R. Demoralization and the longitudinal course of PTSD following Hurricane Mitch. 2012. In press, European Journal of Psychiatry.

39. Schildkraut JJ, Klein DF. The classification and treatment of depressive disorders. In: Shader RI, editor. Manual of Psychiatric Therapeutics: Practical Psychopharmacology \& Psychiatry. Boston, MA: Little, Brown \& Company; 1975. p. 39-61.

40. Klein DF. Endogenomorphic depression. Arch Gen Psychiatry 1974; 31: 447-454. 
41. Klein DF, Gittelman R, Quitkin F, Rifkin A. Diagnosis and Drug Treatment of Psychiatric Disorders: Adults and Children. Baltimore, MD: Williams \& Wilkins; 1980.

42. Clarke DM, Mackinnon AJ, Smith GC, McKenzie DP, Herrman HE. Dimensions of psychopathology in the medically ill. A latent trait analysis. Psychosomatics 2000; 41: 418-425.

43. Clarke DM, Smith GC, Dowe DL, McKenzie DP. An empirically derived taxonomy of common distress syndromes in the medically ill. J Psychosom Res 2003; 54: 323-330.

44. Kendler KS, Gardner CO. Monozygotic twins discordant for major depression: a preliminary exploration of the role of environmental experiences in the aetiology and course of illness. Psychol Med 2001; 31(3): 411-414.

45. Mangelli L, Fava GA, Grandi S, Grassi L, Ottolini F, Percelli $\mathrm{P}$, et al. Assessing demoralization and depression in the setting of medical disease. J Clin Psychiatry 2005; 66(3): 391-394.

46. Porcelli P, Rafanelli C. Criteria for psychosomatic research (DCPR) in the medical setting. Curr Psych Rep 2010; 12: $246-254$.

47. Picardi A, Porcelli P, Pasquini P, Fassone G, Mazzotti $\mathrm{E}$, Lega I, et al. Integration of multiple criteria for psychosomatic assessment of dermatological patients. Psychosomatics 2006; 47: 122-128.

48. Galeazzi GM, Ferrari S, Mackinnon A, Rigatelli M. Interrater reliability, prevalence and relation to ICD-10 diagnoses of the Diagnostic Criteria for Psychosomatic Research in consultation-liaison patients. Psychosomatics 2004; 45: 386-393.

49. Grandi S, Sirri L, Tossani E, Fava GA. Psychological characterization of demoralization in the setting of heart transplantation. J Clin Psychiatry 2011; 72(5): 648-654.

50. Rafanelli C, Roncuzzi R, Milaneschi Y, Tomba E, Colistro MC, Pancaldi LG, et al. Stressful life events, depression and demoralization as risk factors for coronary artery disease. Psychother Psychosom 2005; 74: 179-184.

51. Clarke DM, McLeod JE, Smith GC, Trauer T, Kissane DW. A comparison of psychosocial and physical functioning in patients with motor neuron disease and metastatic cancer. J Palliat Care 2005; 21: 173-179.

52. Porcelli P, De Carne M, Fava GA. Assessing somatization in functional gastrointestinal disorders: Integration of different criteria. Psychother Psychosom 2000; 69: 198-204.

53. Porcelli P, De Carne M, Todarello O. Prediction of treatment outcome of patients with functional gastrointestinal disorders by the Diagnostic Criteria for Psychosomatic Research. Psychother Psychosom 2004; 73: 166-173.

54. Grassi L, Rossi E, Sabato S, Cruciani G, Zambelli M. Diagnostic criteria for psychosomatic research and psy- chosocial variables in breast cancer patients. Psychosomatics 2004; 45: 483-491.

55. Clarke D. Psychological adaptation, demoralization and depression in people with cancer. In: Kissane DW, Maj M, Sartorius N, editors. Depression and Cancer. Oxford: Wiley Blackwell: World Psychiatric Association; 2011. p. 37-50.

56. Grossarth-Maticek R, Bastiaans J, Kanazir DT. Psychosocial factors as strong predictors of mortality from cancer, ischaemic heart disease and stroke: the Yugoslav prospective study. J Psychosom Res 1985; 29: 167-76.

57. Schuitemaker GE, Dinant GJ, van der Pol GA, Appels A. Assessment of vital exhaustion and identification of subjects at increased risk of myocardial infarction in general practice. Psychosomatics 2004; 45: 414-418.

58. Anda R, Williamson D, Jones D, Macera C, Eaker E, Glassman A, et al. Depressed affect, hopelessness, and the risk of ischemic heart disease in a cohort of U.S. adults. Epidemiology 1993; 4: 285-294.

59. Everson SA, Goldberg DE, Kaplan GA, Cohen RD, Pukkala E, Tuomilehto J. Hopelessness and risk of mortality and incidence of myocardial infarction and cancer. Psychosom Med 1996; 58: 113-121.

60. Everson SA, Kaplan GA, Goldberg DE, Salonen JT. Hypertension incidence is predicted by high levels of hopelessness in Finnish men. Hypertension 2000; 35: 561-567.

61. Barefoot JC, Brummett BH, Helms MJ, Mark DB, Siegler IC, Williams RB. Depressive symptoms and survival of patients with coronary artery disease. Psychosom Med 2000; 62: 790-795.

62. Everson SA, Kaplan GA, Goldberg DE, Salonen R, Salonen JT. Hopelessness and 4-year progression of carotid atherosclerosis. The Kuopio Ischemic Heart Disease Risk Factor Study. Arteriosclerosis Thrombosis and Vascular Biology 1997; 17: 1490-1495.

63. Glick ID, Pham D, Davis JM. Concomitant medications may not improve outcome of antipsychotic monotherapy for stabilized patients with nonacute schizophrenia. J Clin Psychiatry 2006; 67: 1261-1265.

64. Papakostas GI, Petersen T, Homberger CH, Green $\mathrm{CH}$, Smith J, Alpert JE, et al. Hopelessness as a predictor of non-response to fluoxetine in major depressive disorder. Ann Clin Psychiatry 2007; 19(1): 5-8.

Corresponding author:

John M. de Figueiredo, MD, ScD

P.O. Box 573 Cheshire,

CT 06410-0573 USA

Tel: 203-272-9628

Fax: 203-272-5124

E-mail: johndefig@ @bcglobal.net 